\title{
Systematic Meta-Analysis of the Association Between a Common NOS1AP Genetic Polymorphism, the QTc Interval, and Sudden Death
}

\author{
Xiaobiao Zang, ${ }^{1 *} \mathrm{MD}$, Sisi $\mathrm{Li},{ }^{2 *} \mathrm{PhD}$, Yonghui Zhao, ${ }^{1} \mathrm{MD}$, Ke Chen, ${ }^{1} \mathrm{MD}$, Xianqing Wang, ${ }^{1} \mathrm{MD}$, \\ Weifeng Song, ${ }^{1} \mathrm{MD}$, Jifang Ma, ${ }^{1} \mathrm{MD}$, Xin Tu, ${ }^{3} \mathrm{PhD}$, Yunlong Xia, ${ }^{4} \mathrm{MD}$, \\ Shulong Zhang, ${ }^{5} \mathrm{MD}$ and Chuanyu Gao, ${ }^{1} \mathrm{MD}$
}

\begin{abstract}
Summary
Contemporary studies have identified rs10494366 in the nitric oxide synthase 1 adaptor protein (NOS1AP) gene as a new genetic marker in modulating the QT interval and sudden cardiac death (SCD) in general populations. However, the conclusions were not coincident. Therefore, we conducted for the first time a system evaluation of the relativity of rs10494366, the QT interval, and sudden death by meta-analysis. In our study, the meta-analysis displayed the GG genotype of rs10494366 correlated with the QT interval in women with no heterogeneity, and in diabetes mellitus (DM) patients with minor heterogeneity. In the Caucasian population, the correlation of rs10494366 and sudden death was significant. The heterogeneity referred to the relevance between rs10494366 and sudden death in the Asian population. In conclusion, the minor allele of rs10494366 may have an impact on the QT interval in women or DM patients and may have a potential role in sudden death in the Caucasian population.
\end{abstract}

Key words: Sudden cardiac death, Single nucleotide polymorphism

(Int Heart J 2019; 60: 1083-1090)

$\mathrm{S}$ udden death is the leading cause of mortality in developed countries, and accordingly an important public health concern. Sudden death is mostly cardiovascular and arrhythmic in origin, with ventricular fibrillation as the ultimate precipitating mechanism of cardiac arrest. The mechanism of sudden death is complex, and currently not completely understood. Many clinical and genetic indicators have been identified and helped in distinguishing high-risk patients in previous studies. In contemporary studies, rs10494366 in the nitric oxide synthase 1 adaptor protein (NOS1AP) gene was identified as a novel genetic marker in modulating the QT interval and sudden cardiac death (SCD) in the general population. ${ }^{1-3)}$ However, the conclusions of the studies were not coincident, ${ }^{4,5}$ and meta-analysis was considered.

\section{Methods}

Databases, including PubMed, Embase, ScienceDirect, Google Scholar, WanFang, and CNKI were searched to identify relevant studies up to August 2018. The meta- analysis included two components: the association of rs 10494366 and the QT interval, as well as the association of rs10494366 and sudden death. The following terms were included in the search: "rs10494366" and "polymorphism or variant" and "nitric oxide synthase 1 adaptor protein or NOS1AP" and "QT interval or QT or QT prolongation" for the meta-analysis involving the QT interval and rs10494366. The inclusion criteria were as follows: 1) studies were in Hardy-Weinberg equilibrium (HWE) and performed in sporadic population; 2) studies that provided data involving the correlation coefficient and the 95\% confidence interval (CI) or standard error for linear correlation of the QTc interval and rs10494366. Studies in which patients were enrolled with one or more mutation sites were excluded from the analysis. Studies were also excluded if the correction of the QT (QTc) interval did not use the Bazett's formula $(\mathrm{QTc}=\mathrm{QT} / \sqrt{R R})$. The following search terms were used for the meta-analysis of sudden death and rs10494366: "nitric oxide synthase 1 adaptor protein or NOS1AP" and "polymorphism or variant" and "heart arrest or SCD or sudden arrhythmic death

From the 'Zhengzhou University People's Hospital, Fuwai Central China Cardiovascular Hospital, Zhengzhou University, Zhengzhou, China, ${ }^{2}$ Gannan Medical University, Jiangxi, China, ${ }^{3}$ Key Laboratory of Molecular Biophysics of the Ministry of Education, College of Life Science and Technology and Center for Human Genome Research, Cardio-X Institute, Huazhong University of Science and Technology, Wuhan, China, ${ }^{4}$ First Affiliated Hospital of Dalian Medical University, Dalian, China and ${ }^{5}$ Department of Cardiology, Affiliated Zhongshan Hospital of Dalian University, Dalian, China.

*These authors contributed equally to this work.

This study was supported by the Medical Science and Technique Program of He'nan Provincial Health and Family Planning Commission (No. 201602214); Science and Technique Program of He'nan Provincial Department of Science and Technology(NO:172102310065).

Address for correspondence: Chuanyu Gao, MD, Zhengzhou University People's Hospital, Fuwai Central China Cardiovascular Hospital, Zhengzhou University, No. 1 Fuwai Avenue, Zhengzhou, China. E-mail: gaochuanyu01@163.com

Received for publication January 13, 2019. Revised and accepted March 26, 2019.

Released in advance online on J-STAGE August 23, 2019.

doi: 10.1536/ihj.19-024

All rights reserved by the International Heart Journal Association. 


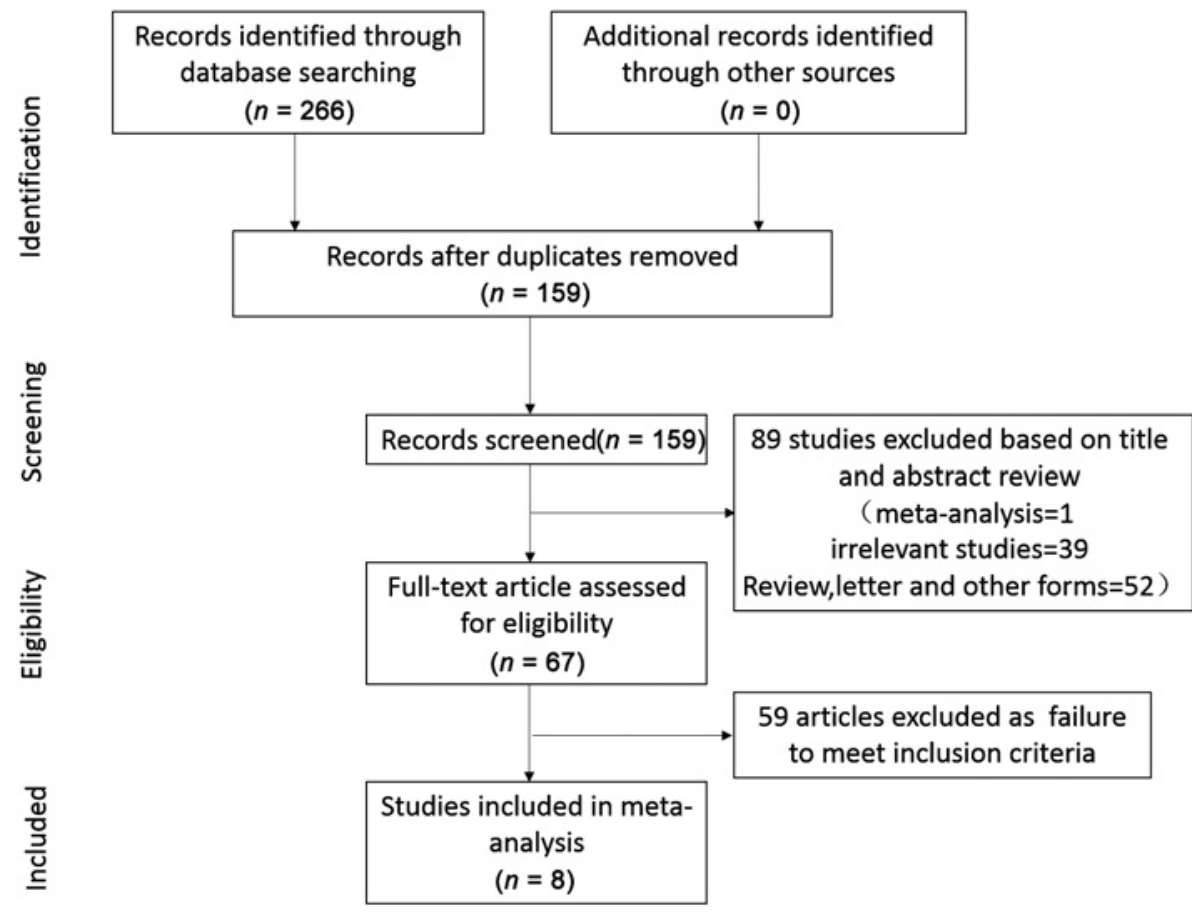

Figure 1. Flowchart of the study selection process of the meta-analysis of the association between rs 10494366 and the QT interval.

or sudden cardiac arrest or sudden death." The inclusion criteria were as follows: 1) case-control studies focused on the association between NOS1AP and sudden death; 2) studies that provided sufficient genotype data (TT, TG, and GG) in cases as well as control groups; and 3) all death events had definitive diagnostic criteria. Exclusion criteria were as follows: 1) no detailed genotype frequencies and 2) rs10494366 was deviated from HWE in the control group. For the two meta-analyses in this study, the abstracts, reviews, simple commentaries, editorials, case reports, and meta-analyses were excluded. When overlapping studies were obtained, the study with the largest sample size was included. If data in a selected study was lacking, the authors were contacted to see if more information could be obtained. Only studies published in the English or Chinese language were included.

Two reviewers independently extracted data, and any disagreement was resolved by joint review until a consensus was reached. For the association of the QT interval and rs10494366, author, publication year, country, ethnicity, study name, numbers of all subjects and each genotype, QT interval or QTc interval of all subjects and each genotype, minor allele frequency (MAF) in the entire group or subgroup, linear regression coefficient $(\beta)$, and standard error (SE) were extracted from candidate studies. Simultaneously, in the meta-analysis of rs10494366 and sudden death, author, publication year, country, ethnicity, MAF in controls, related diseases, and the distribution of three genotypes in the case and control groups were extracted. The Newcastle-Ottawa Quality Assessment Scale (NOS) was used independently by two authors to evaluate the quality of the included studies (http://www.ohri.ca/pro grams/clinical_epidemiology/oxford.asp). Studies with a score of seven stars or greater were considered of high quality and were included in the current meta-analysis.

In this study, pooled effect size $\beta$ and SE were used to determine the strength of associations between rs 10494366 and QT interval under an additive genetic model. $\beta$ was pooled using a random-effect model if heterogeneity existed, otherwise, the fixed model was conducted. Analysis of the correlation between rs10494366 and SCD was implemented by ORs with $95 \%$ CIs in four genetic models. The heterogeneity Q-test and I-squared test were used to assess the heterogeneity between studies. $P<0.05$ and I-squared $\geq 50 \%$ were considered of significant heterogeneity. Subgroup analysis was performed to explore the sources of heterogeneity. Publication bias was calculated using Begg's funnel plot or Egger's plot, and the significance level for both was set at 0.05. Metaanalysis was performed using STATA 12.0 (STATA Corp, College Station, TX, USA). $P$-value $<0.05$ was considered statistically significant.

\section{Results}

For the meta-analysis of rs10494366 and the QTc interval, a total of 266 studies were selected through a database search. A total of eight eligible studies were enrolled after removing duplicates, screening by title and abstract review, and assessment of eligibility criteria (Figure 1). The total number of subjects in the present study is 45,206, which included 27,556 Caucasian, 14,715 African, 2436 Asian, and 499 Hispanics. ${ }^{1,6-12)}$ Five studies consisted of more than one study cohort (Table I). 


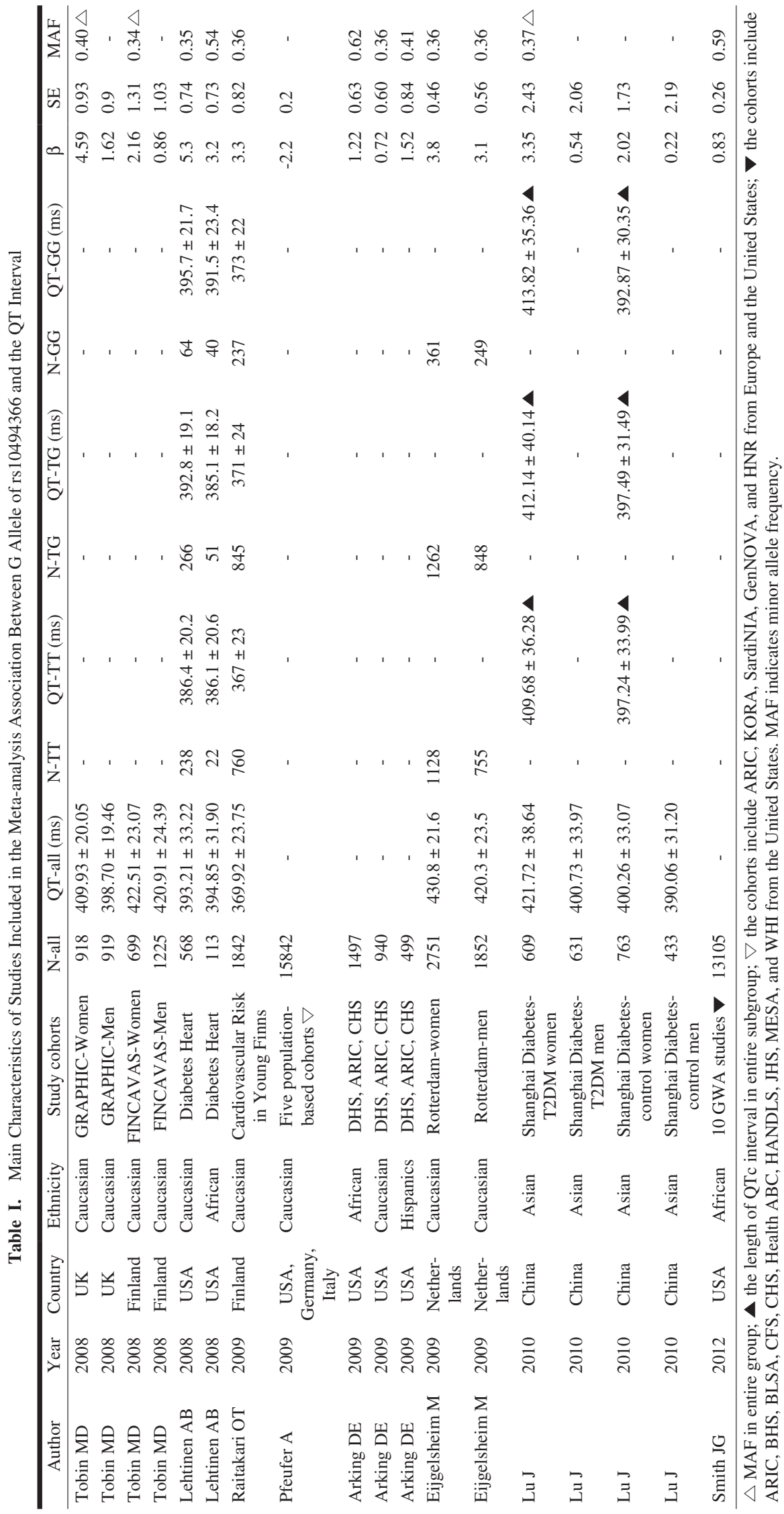




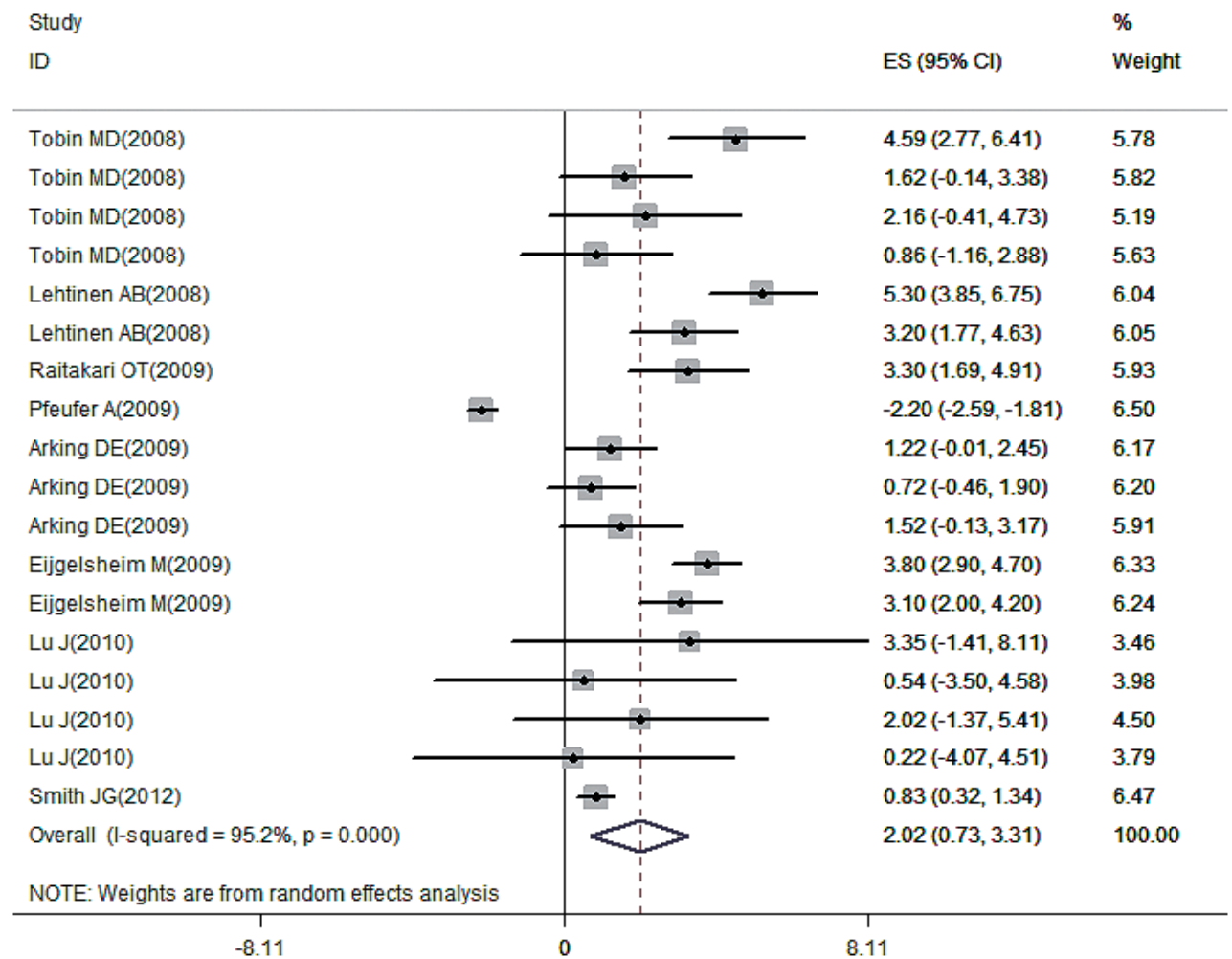

Figure 2. Forest plots for the association between rs10494366 and the QT interval in all groups using the additive genetic model.

Table II. Subgroup Meta-analysis of the Association Between rs10494366 and the QT Interval

\begin{tabular}{lrrrrc}
\hline & $\begin{array}{c}\text { Study } \\
\text { number }\end{array}$ & $\begin{array}{r}\text { Heterogeneity } \\
\text { statistic }\end{array}$ & I-squared & $\mathrm{Z}$ & $P$ \\
\hline Gender & & & & & \\
$\quad$ Men & 5 & 6.16 & $35.0 \%$ & 3.13 & 0.002 \\
Women & 5 & 3.31 & $0.0 \%$ & 9.76 & 0.000 \\
$\quad$ Mixed & 8 & 222.07 & $96.8 \%$ & 1.85 & 0.064 \\
Subjects number & & & & & \\
$\quad$ 1000 & 11 & 33.65 & $70.3 \%$ & 4.19 & 0.000 \\
$>1000$ & 7 & 250.02 & $97.6 \%$ & 1.52 & 0.130 \\
Age (years) & & & & & \\
$<45$ & 9 & 162.98 & $95.1 \%$ & 1.66 & 0.097 \\
$\quad$ 45 & 9 & 38.56 & $79.3 \%$ & 4.72 & 0.000 \\
Diseases & & & & & \\
DM & 4 & 7.18 & $58.2 \%$ & 3.93 & 0.000 \\
Others & 14 & 286.23 & $95.5 \%$ & 2.42 & 0.015 \\
Study design & & & & & \\
PB & 12 & 283.23 & $96.1 \%$ & 2.26 & 0.024 \\
HB & 6 & 15.48 & $67.7 \%$ & 3.35 & 0.001 \\
Ethnicity & & & & & \\
Caucasian & 10 & 319.80 & $97.2 \%$ & 2.10 & 0.035 \\
Asian & 4 & 1.23 & $0.0 \%$ & 1.45 & 0.146 \\
African & 3 & 9.39 & $78.7 \%$ & 2.45 & 0.014 \\
Hispanics & 1 & 0.00 & - & 1.81 & 0.070 \\
\hline Indics & & & &
\end{tabular}

DM indicates diabetes mellitus; $\mathrm{HB}$, hospital-based; and $\mathrm{PB}$, population-based.

Figure 2 shows the results of the random-effect model combining the $\beta$ for QTc interval. Among the 18 studies, seven showed a significant positive relation between SNP rs10494366 and QTc interval. For the correlation, the pooled effect size $\beta$ was 3.07 , but with a huge heterogeneity in statistics $\left(Q=350.87, I^{2}=95.2 \%\right)$. Subsequently, meta-analysis of effects in the subgroup was performed, and the results are shown in Table II. The subgroup analyses included gender, subject number, age, diseases, study design, and ethnicity. There were five cohorts that focused on gender differences. The pooled effect size was 9.76 for the women cohort in which no heterogeneity emerged $\left(\mathrm{Q}=3.31, \mathrm{I}^{2}=0.0 \%\right)$. The $\mathrm{I}^{2}$ was $35.5 \%$ and $96.8 \%$, respectively, for men and mixed individuals. All studies were divided into two subgroups based on whether the number was greater than 1000 , and all showed significant heterogeneity. Similarly, when studies were divided into two subgroups based on whether the age was $>45$ years, whether subjects were combined with DM, and the type of study design, all pooled effect sizes showed heterogeneity. The association of rs10494366 and the QT interval in DM patients was significant with heterogeneity statistic equaling $7.18(P=0.066)$ and $\mathrm{I}^{2}=58.2 \%$. In the Asian population, the pooled effect size was not significant, with a $\mathrm{z}$-value being 1.45. However, in Caucasian and African populations, the associations were significant but with a huge heterogeneity. Only one case focused on Hispanics and the results indicated a negative association. The Begg's and Egger's tests were conducted for women and DM subgroups, respectively (Supplemental Figures 1, 2 ), and our data showed that there was no publication bias in these two subgroups. 


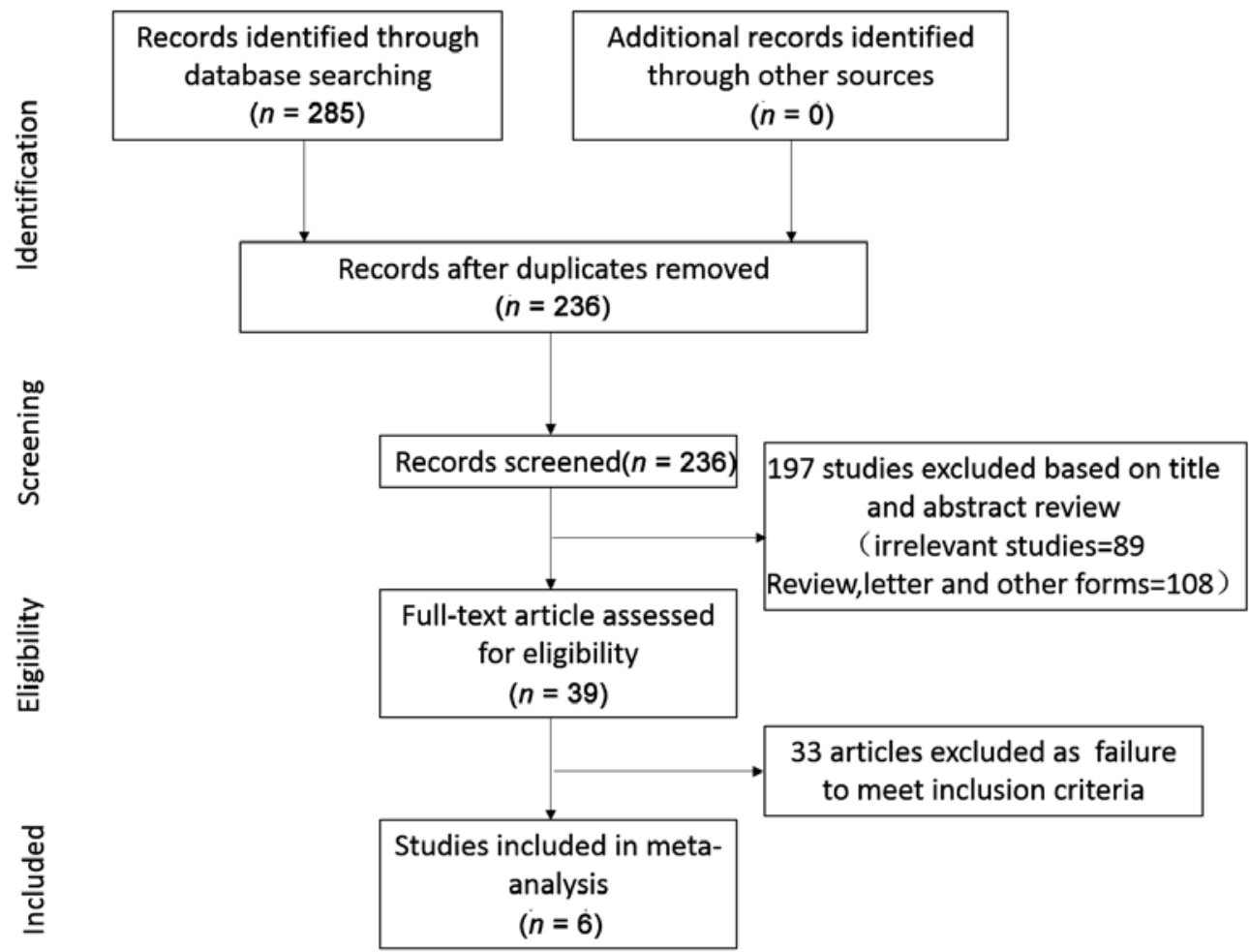

Figure 3. Flowchart of the study selection process in the meta-analysis of the association between rs 10494366 and sudden death.

Table III. Main Characteristics of Studies Included in the Meta-analysis of rs 10494366 and Sudden Death

\begin{tabular}{|c|c|c|c|c|c|c|c|c|c|c|c|}
\hline \multirow{2}{*}{ Author } & \multirow{2}{*}{ Year } & \multirow{2}{*}{ Country } & \multirow{2}{*}{ Ethnicity } & \multicolumn{3}{|c|}{ Case } & \multicolumn{3}{|c|}{ Control } & \multirow{2}{*}{ MAF } & \multirow{2}{*}{ Related diseases } \\
\hline & & & & GG & GT & TT & GG & GT & TT & & \\
\hline Osawa M & 2009 & Japan & Asian & 16 & 18 & 8 & 99 & 97 & 14 & 0.298 & Sudden infant death \\
\hline Becker ML & 2009 & Netherlands & Caucasian & 12 & 27 & 15 & 134 & 473 & 452 & 0.350 & Cardiovascular mortality \\
\hline Huang L & 2014 & China & Asian & 65 & 46 & 12 & 74 & 67 & 25 & 0.352 & SUNDS \\
\hline Huang JL & 2014 & China & Asian & 30 & 18 & 12 & 33 & 27 & 20 & 0.419 & Sudden unexpected death \\
\hline Earle $\mathrm{N}$ & 2014 & New Zealand & Caucasian & 8 & 9 & 4 & 30 & 106 & 63 & 0.417 & Resuscitated SCD/SCD \\
\hline Marcsa B & 2015 & Hungary & Caucasian & 50 & 160 & 132 & 40 & 129 & 110 & 0.375 & Cardiac death \\
\hline
\end{tabular}

MAF indicates minor allele frequency; SCD, sudden cardiac death; and SUNDS, sudden unexplained nocturnal death syndrome.

In the meta-analysis of association between rs 10494366 and sudden death, a total of 285 articles were initially selected based on the search strategy. After screening and assessment (49 duplicates, 197 studies excluded based on title and abstract review, 33 articles excluded due to failure to meet the inclusion criteria), a total of six studies were included ${ }^{3,13-17)}$ (Figure 3). Characteristics of the included studies are presented in Table III. Three studies were based on the Caucasian population. The related diseases included sudden infant death, sudden cardiovascular mortality, sudden unexplained nocturnal death syndrome, sudden unexpected death, resuscitated $\mathrm{SCD} / \mathrm{SCD}$, and cardiac death.

In the forest plot stratified by ethnicity (Figure 4), the association of rs10494366 $\mathrm{G}$ allele and sudden death was significant in the Caucasian population, however, slight heterogeneity existed $\left(\mathrm{Q}=4.86, \mathrm{I}^{2}=58.9 \%\right)$, which assumed an allele model. The dominant model did not show significance without obvious heterogeneity in Cau- casians $\left(\mathrm{Q}=2.24, \mathrm{I}^{2}=10.6 \%\right)$. In the Caucasian population, the OR was $1.19(0.70-1.67)$ with $\mathrm{z}=4.80, P<$ 0.001 , thereby assuming a dominant model. Analogous results appeared in a recessive model with $\mathrm{Q}=2.94, \mathrm{I}^{2}=$ $31.9 \%$ in Caucasians and $\mathrm{Q}=2.86, \mathrm{I}^{2}=30.1 \%$ in the Asian population. The associations were significant in both the Asian and Caucasian populations with $\mathrm{OR}=1.40$ ( $\mathrm{z}=3.09, P=0.002)$ and $1.04(\mathrm{z}=3.40, P=0.001)$, respectively. No heterogeneity was observed in Caucasians for the homozygote model and $\mathrm{OR}=1.46(\mathrm{z}=2.43, P=$ 0.015). However, in the Asian population, the heterogeneity was significant in the dominant, recessive, and homozygote model. The pooled OR was $1.10(\mathrm{z}=5.47, P<$ $0.001)$ in the overall population without homozygosity in the recessive model (Supplemental Table). No statistically significant data was observed by Begg's and Egger's tests in all four genotype models (Supplemental Table). 


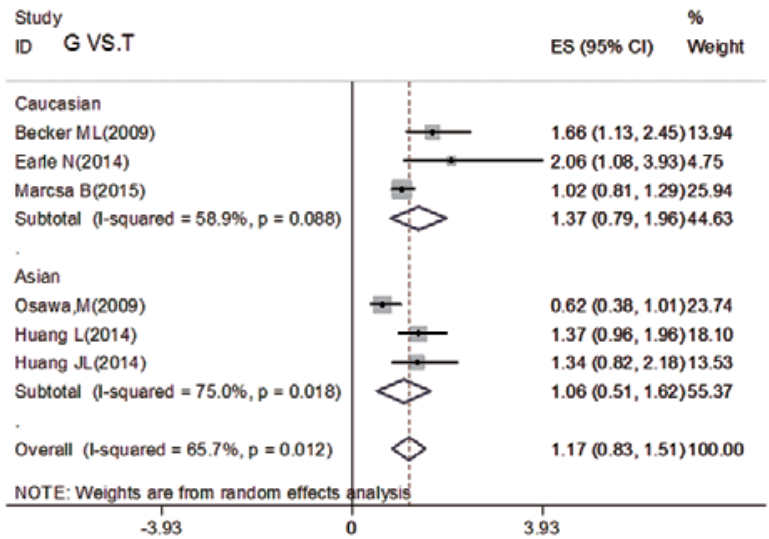

\begin{tabular}{|c|c|c|c|}
\hline Study & & & $\%$ \\
\hline ID GG+GT VS.TT & & $\mathrm{ES}(95 \% \mathrm{Cl})$ & Weight \\
\hline Caucasian & & & \\
\hline Becker ML(2009) & & $1.94(1.05,3.56)$ & ) 11.32 \\
\hline Earle N(2014) & & $1.97(0.64,6.09)$ & 3.40 \\
\hline Marcsa B(2015) & \pm & $1.04(0.75,1.43)$ & 26.53 \\
\hline Subtotal (1-squared $=10.6 \%, p=0.327$ ) & & $1.19(0.70,1.67)$ & 41.26 \\
\hline . & & & \\
\hline Asian & & & \\
\hline Osawa,M(2009) & \pm & $0.30(0.12,0.78)$ & 26.73 \\
\hline Huang L(2014) & & $1.39(0.87,2.22)$ & 20.16 \\
\hline Huang JL(2014) & & $1.33(0.59,3.00)$ & 11.85 \\
\hline Subtotal (1-squared $=79.6 \%, p=0.007)$ & & $0.92(0.06,1.79)$ & 58.74 \\
\hline . & & & \\
\hline Overall (l-squared $=72.6 \%, p=0.003$ ) & & $1.08(0.55,1.61)$ & 1) 100.00 \\
\hline NOTE: Weights are from random effects & alysi & & \\
\hline
\end{tabular}

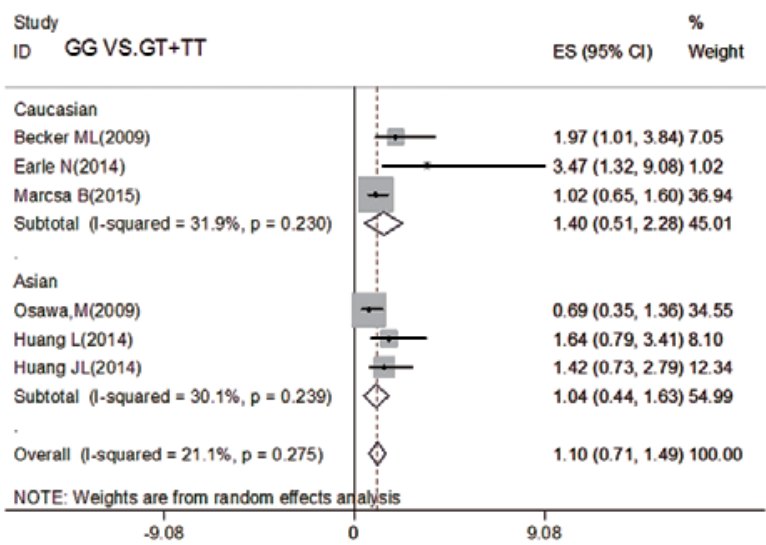

\begin{tabular}{|c|c|c|c|}
\hline \multicolumn{3}{|l|}{ Study } & \multirow{2}{*}{$\begin{array}{l}\% \\
\text { Weight }\end{array}$} \\
\hline ID GG VS.TT & & $\mathrm{ES}(95 \% \mathrm{Cl})$ & \\
\hline \multicolumn{4}{|l|}{ Caucasian } \\
\hline Becker ML(2009) & $=$ & $2.70(1.23,5.91)$ & 7.31 \\
\hline Earle N(2014) & & $4.20(1.17,15.05)$ & 1.01 \\
\hline Marcsa B(2015) & - & $1.04(0.64,1.70)$ & 30.49 \\
\hline Subtotal $(1-$ squared $=22.7 \%, p=0.274)$ & $\infty$ & $1.46(0.28,2.64)$ & 38.81 \\
\hline \multicolumn{4}{|l|}{. } \\
\hline \multicolumn{4}{|l|}{ Asian } \\
\hline Osawa,M(2009) & - & $0.28(0.10,0.78)$ & 33.85 \\
\hline Huang L(2014) & $\rightarrow$ & $1.83(0.85,3.93)$ & 13.39 \\
\hline Huang JL(2014) & $\frac{1}{2}$ & $1.51(0.63,3.62)$ & 13.95 \\
\hline Subtotal (1-squared $=66.3 \%, p=0.051$ ) & $\$$ & $1.00(-0.11,2.11)$ & 61.19 \\
\hline$\cdot$ & 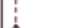 & & \\
\hline Overall $(1-$ squared $=64.6 \%, p=0.015)$ & 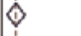 & $1.11(0.40,1.82)$ & 100.00 \\
\hline NOTE: Weights are from random effects & ysis & & \\
\hline
\end{tabular}

Figure 4. Forest plots for four models of the association between rs10494366 and sudden death stratified by ethnicity.

\section{Discussion}

Recently, many studies revealed that common variants in the NOS1AP gene were associated with QTc interval variation in several large population samples. The NOS1AP gene which encodes the nitric oxide synthase 1 adaptor protein has been demonstrated to regulate neuronal nitric oxide synthase activation, and enhanced Dexras1 activation. ${ }^{18)}$ Neuronal nitric oxide synthase protein expression has been found in cardiac myocytes, and may play a role in controlling basal and adrenergically stimulated cardiac contractility, as well as in autonomic control of the heart rate. ${ }^{19,20)}$ Overexpression of neuronal nitric oxide synthase protein results in shortening of the cardiac action potential, a decrease in L-type $\mathrm{Ca}^{2+}$ current, and a smaller increase in the delayed rectifier potassium current, resulting in prolongation of the QTc interval. ${ }^{21)}$ In previous studies, it has been demonstrated that rs 10494366, which is located in the intron region of NOS1AP, was associated with the QT interval and sudden death in independent populations. ${ }^{1,6-12)}$ However, the conclusions were inconsistent. ${ }^{4,5)}$ SNP rs10494366 may influence the QT interval in women and DM patients as revealed by the present metaanalysis. Furthermore, our meta-analysis also indicated an association between rs10494366 and sudden death in the Caucasian population.

The QT interval, which represents the electrocar- diographic correlation of ventricular depolarization and repolarization, is susceptible to many factors. In the QT interval, approximately $35 \%$ of the variation duration is heritable, and a majority of identified SNPs implicate gene regulatory dysfunction in QT prolongation. ${ }^{22,23)}$ As revealed by our meta-analysis, the GG genotype of rs 10494366 correlated with the QT interval in women in whom no heterogeneity is found, and in DM patients with slight heterogeneity. It has long been recognized that the QTc, which is corrected by the heart rate, is of a longer duration in women when compared to men, whereas such gender differences are not observed neonates and older children. ${ }^{24)}$ In men, testosterone may contribute to QT interval shortening by modifying ion channels, specifically potassium and calcium channels, ${ }^{25)}$ whereas estradiol may have an acute dose-dependent blocking effect on the potassium channel, which leads to QT prolongation. ${ }^{26)}$ Therefore, it is reasonable to speculate that rs10494366 may share a common pathway with estradiol, which possibly influences ion channels.

DM patients are usually complicated with QT prolongation, which is considered a specific sign of autonomic cardiac dysfunction, and a high mortality risk. However, regardless of the fact whether DM is complicated with autonomic neuropathy, several general abnormalities, including QT prolongation in the ECG have been repeatedly reported in DM patients. Although DM has proven to pro- 
mote a significant prolongation in the QTc interval in streptozotocin-induced diabetes in rats, data that focus on DM patients were a little bit controversial. ${ }^{27)}$ The fact that genetic variants in NOS1AP reproducibly alter the QT interval duration in families, aggregating DM demonstrated that genetic effects still play an important role regardless of the strong effect of diabetes on repolarization. ${ }^{7}$ The present meta-analysis showed a significant association between rs10494366 and the QTc interval in DM patients with a z-value of 3.93 , which assuredly added a new genetic biological predictor for QTc interval in DM although the mechanism between rs10494366 and the QTc interval in DM patients is still not clear.

As described in previous studies, abnormal cardiac repolarization plays an important role in rapid ventricular arrhythmia, which results in SCD. ${ }^{28}$ Therefore, in this study, we conducted meta-analysis on the relation between rs10494366 and sudden death. Our data showed an association between rs10494366 and sudden death in the Asian population as well as in the overall population that was ambiguous due to the heterogeneity or negative conclusion that existed in the pooled studies. However, the correlation was significant in the Caucasian population without significant heterogeneity. Although associations have been shown between NOS1AP SNPs and the QTc interval, this is not yet known for the effect of the same SNPs on the risk of cardiac events. ${ }^{2,29)}$ On the other hand, several studies showed that NOS1AP had a distinct association with cardiac events and SCD, but not with the QTc. $^{30)}$

Three studies focused on the Asian population in the present meta-analysis on SCD. Osawa, et al. ${ }^{13)}$ demonstrated that the $\mathrm{T}$ allele of rs10494366 may be associated with the occurrence of sudden infant death syndrome (SIDS) in a manner of recessive inheritance. One of the speculated causes for SIDS is hereditary disease, in which a long QT in the electrocardiogram has been investigated in view of the mutations in various genes that represent ion channels. In two studies ${ }^{15,16)}$ that were performed in China, a negative conclusion was observed in patients with sudden unexplained nocturnal death syndrome or sudden unexpected death, however, the number of subjects was limited. Becker, et al. ${ }^{14)}$ studied 1113 participants in the Rotterdam study, and investigated whether this polymorphism was associated with cardiovascular mortality in users of calcium channel blockers. The data revealed that the $\mathrm{G}$ allele of rs10494366 in the NOS1AP gene was associated with increased all-cause and cardiovascular mortality in the Caucasian population. In another study, ${ }^{3)}$ a total of 273 patients with LQTS were included from the New Zealand Cardiac Inherited Disease Registry, among which 31 patients had experienced death or resuscitated SCD (RSCD). The study showed that modifier SNPs including rs10494366 in NOS1AP were associated with an increased risk of SCD and cardiac events in patients with LQTS. However, in a study that aimed to identify a genetic polymorphism that may potentially be used as a universal predictor of cardiac death risk in Caucasian, rs 10494366 did not show significance. ${ }^{17)}$

Our meta-analyses have the following limitations: firstly, the QT interval duration is a complex trait with many environmental and genetic determinants. Multiple factors can influence the QT interval, which displayed distinct results in enrolled studies. Although strict filter criteria were set, detailed subgroup analysis was performed, heterogeneity of enrolled studies cannot be avoided. Secondly, the number of enrolled studies was too small to provide sufficient verification for the association of this SNP and sudden death. Moreover, studies that focused on the general population are scarce. Thirdly, data directly obtained from SCD subjects are of utmost importance although SCD is a main form of different types of sudden death. At last, the mechanism underlying the current association is unknown. Rs10494366, located in intron 1 of NOS1AP, does not have a known biological function, thereby suggesting that a causal untyped variant that correlated with rs10494366 may exist.

Nevertheless, the present first coherent meta-analysis, which involves an association between rs10494366, the QT interval, and sudden death, suggested that rs10494366 may have impact on the QT interval in women or DM patients, and have a potential in clinical risk stratification for sudden death. Obviously, additional studies on the association of rs10494366 and sudden death are warranted, and the underlying biological and electrophysiological mechanism need to be revealed.

\section{Disclosures}

Conflicts of interest: The authors declare no conflicts of interest.

\section{References}

1. Arking DE, Pfeufer A, Post W, et al. A common genetic variant in the NOS1 regulator NOS1AP modulates cardiac repolarization. Nat Genet 2006; 38: 644-51.

2. Kao WH, Arking DE, Post W, et al. Genetic variations in nitric oxide synthase 1 adaptor protein are associated with sudden cardiac death in US white community-based populations. Circulation 2009; 119: 940-51.

3. Earle N, Yeo HD, Pilbrow A, et al. Single nucleotide polymorphisms in arrhythmia genes modify the risk of cardiac events and sudden death in long QT syndrome. Heart Rhythm 2014; 11: 76-82.

4. Nolte IM, Wallace C, Newhouse SJ, et al. Common genetic variation near the phospholamban gene is associated with cardiac repolarisation: Meta-Analysis of three genome-wide association studies. PLoS One 2009; 4: e6138.

5. Jamshidi Y, Nolte IM, Dalageorgou C, et al. Common variation in the NOS1AP gene is associated with drug-induced QT prolongation and ventricular arrhythmia. J Am Coll Cardiol 2012; 60: $841-50$.

6. Tobin MD, Kahonen M, Braund $\mathrm{P}$, et al. Gender and effects of a common genetic variant in the NOS1 regulator NOS1AP on cardiac repolarization in 3761 individuals from two independent populations. Int J Epidemiol 2008; 37: 1132-41.

7. Lehtinen AB, Newton-Cheh C, Ziegler JT, et al. Association of NOS1AP genetic variants with QT interval duration in families from the Diabetes Heart Study. Diabetes 2008; 57: 1108-14.

8. Raitakari OT, Blom-Nyholm J, Koskinen TA, Kahonen M, Viikari JS, Lehtimaki T. Common variation in NOS1AP and $\mathrm{KCNH} 2$ genes and QT interval duration in young adults. The Cardiovascular Risk in Young Finns Study. Ann Med 2009; 41: 144-51. 
9. Pfeufer A, Sanna S, Arking DE, et al. Common variants at ten loci modulate the QT interval duration in the QTSCD Study. Nat Genet 2009; 41: 407-14.

10. Eijgelsheim M, Aarnoudse AL, Rivadeneira F, et al. Identification of a common variant at the NOS1AP locus strongly associated to QT-interval duration. Hum Mol Genet 2009; 18: 347-57.

11. $\mathrm{Lu} \mathrm{J}, \mathrm{Hu} \mathrm{C}, \mathrm{Hu} \mathrm{W}$, et al. A common variant of NOS1AP is associated with QT interval duration in a Chinese population with Type 2 diabetes. Diabet Med 2010; 27: 1074-9.

12. Smith JG, Avery CL, Evans DS, et al. Impact of ancestry and common genetic variants on QT interval in African Americans. Circ Cardiovasc Genet 2012; 5: 647-55.

13. Osawa M, Kimura R, Hasegawa I, Mukasa N, Satoh F. SNP association and sequence analysis of the NOS1AP gene in SIDS. Leg Med (Tokyo) 2009; 11: S307-8.

14. Becker ML, Visser LE, Newton-Cheh C, et al. A common NOS $1 \mathrm{AP}$ genetic polymorphism is associated with increased cardiovascular mortality in users of dihydropyridine calcium channel blockers. Br J Clin Pharmacol 2009; 67: 61-7.

15. Huang L, Yu Y, Chen Y, et al. Association of common variants in NOS1AP gene with sudden unexplained nocturnal death syndrome in the southern Chinese Han population. Int J Legal Med 2014; 128: 933-8.

16. Huang JL, Hao B, Wang XG, et al. Correlation of NOS1AP gene polymorphisms with sudden unexpected death in Chinese Han population. Fa Yi Xue Za Zhi 2014; 30: 27-30, 35.

17. Marcsa B, Denes R, Voros K, et al. A common polymorphism of the human cardiac sodium channel alpha subunit (SCN5A) gene is associated with sudden cardiac death in chronic ischemic heart disease. PLoS One 2015; 10: e0132137.

18. Fang M, Jaffrey SR, Sawa A, Ye K, Luo X, Snyder SH. Dexras 1: A G protein specifically coupled to neuronal nitric oxide synthase via CAPON. Neuron 2000; 28: 183-93.

19. Sears CE, Ashley EA, Casadei B. Nitric oxide control of cardiac function: Is neuronal nitric oxide synthase a key component? Philos Trans R Soc Lond B Biol Sci 2004; 359: 1021-44.

20. Sugiyama K, Sasano T, Kurokawa J, et al. Oxidative stress induced ventricular arrhythmia and impairment of cardiac function in Noslap deleted mice. Int Heart J 2016; 57: 341-9.

21. Chang KC, Barth AS, Sasano T, et al. CAPON modulates car- diac repolarization via neuronal nitric oxide synthase signaling in the heart. Proc Natl Acad Sci U S A 2008; 105: 4477-82.

22. Newton-Cheh C, Larson MG, Corey DC, et al. QT interval is a heritable quantitative trait with evidence of linkage to chromosome 3 in a genome-wide linkage analysis: The Framingham Heart Study. Heart Rhythm 2005; 2: 277-84.

23. Avery CL, Wassel CL, Richard MA, et al. Fine mapping of QT interval regions in global populations refines previously identified QT interval loci and identifies signals unique to African and Hispanic descent populations. Heart Rhythm 2017; 14: 57280 .

24. Rautaharju PM, Zhou SH, Wong S, et al. Sex differences in the evolution of the electrocardiographic QT interval with age. Can J Cardiol 1992; 8: 690-5.

25. Bai CX, Kurokawa J, Tamagawa M, Nakaya H, Furukawa T. Nontranscriptional regulation of cardiac repolarization currents by testosterone. Circulation 2005; 112: 1701-10.

26. Lehmann MH, Timothy KW, Frankovich D, et al. Age-gender influence on the rate-corrected QT interval and the QT-heart rate relation in families with genotypically characterized long QT syndrome. J Am Coll Cardiol 1997; 29: 93-9.

27. Kittnar O. Electrocardiographic changes in diabetes mellitus. Physiol Res 2015; 64: S559-66.

28. Tse G, Yan BP. Traditional and novel electrocardiographic conduction and repolarization markers of sudden cardiac death. Europace 2017; 19: 712-21.

29. Aarnoudse AJ, Newton-Cheh C, de Bakker PI, et al. Common NOS1AP variants are associated with a prolonged QTc interval in the Rotterdam Study. Circulation 2007; 116: 10-6.

30. Earle NJ, Poppe KK, Pilbrow AP, et al. Genetic markers of repolarization and arrhythmic events after acute coronary syndromes. Am Heart J 2015; 169: 579-86.

\section{Supplemental Files}

Supplemental Table

Supplemental Figures 1, 2

Please see supplemental files; https://doi.org/10.1536/ihj.19-024 